\title{
Predictors of Coronary Slow Flow Phenomenon: A Retrospective Study
}

\author{
Satish Kumar Rao V. ${ }^{1} \quad$ Indrani Garre ${ }^{1}$ \\ ${ }^{1}$ Department of Cardiology, Nizam's Institute of Medical Sciences, \\ Hyderabad, Telangana, India
}

Address for correspondence Satish Kumar Rao V., MD, DTCD, DM, Department of Cardiology, Nizam's Institute of Medical Sciences (NIMS), Hyderabad, Telangana 500082, India (e-mail: drsatishrao09@gmail.com).

Ind J Car Dis Wom 2019;4:85-91

\begin{abstract}
Keywords

- coronary angiogram

- slow flow

- BMI

$-D M$

- smoking

Aim This study aimed to analyze laboratory predictors, angiographic profile, clinical profile, and risk factors for coronary slow flow (CSF) phenomenon without coronary obstructive lesion in patients who came for a coronary angiogram.

Materials and Methods The case-control study consisted of patients who underwent coronary angiography and were divided into two groups: patients with coronary slow flow (case group, $n=100$ ) and patients with the normal coronary flow (control group, $n=100$ ). Coronary flow was studied using corrected thrombolysis in myocardial infarction frame count (CTFC). The slow flow was defined as CTFC beyond 2 standard deviations from the normal published range. Risk factors including age, sex, diabetes mellitus (DM), hypertension, dyslipidemia, smoking, body mass index (BMI), hematological and biochemical parameters (complete blood picture, platelet count, total and differential leucocyte count, platelet-to-lymphocyte ratio [PLR], neutrophil-to-lymphocyte ratio [NLR] and lipid profile) were assessed. In both groups, clinical information was collected, and laboratory parameters were measured and compared. Results Patients with CSF were more likely to be male and active smokers. Total cholesterol, triglyceride, BMI, and DM were more commonly seen in the CSF group compared with the control group. Inflammatory markers like uric acid $(p=0.03)$ and high-sensitivity C-reactive protein (Hs-CRP) $(p=0.000)$ were found to be statistically significant. Hematocrit $(p=0.023)$, NLR $(p=0.001)$, total cholesterol $(p=0.000)$, triglycerides $(p=0.000)$, and BMI $(p=0.000)$ were statistically significant. PLR has the tendency of statistically significance $(p=0.059)$. BMI, total cholesterol, triglycerides, and Hs-CRP were strong predictors for CSF.

Conclusion CSF was common in males, smokers, DM patients, and it was associated with high NLR, uric acid, and Hs-CRP levels. The independent predictor of CSF was BMI, total cholesterol, triglycerides, and Hs-CRP levels. These findings provide an impetus for additional studies to confirm the role of other inflammatory markers in CSF patients and treatment strategies depending on that.
\end{abstract}

\section{Introduction}

Coronary slow flow (CSF) is a common finding in the coronary angiographic studies. CSF phenomenon is characterized by delayed coronary vessel opacification of the distal segment in the absence of significant epicardial coronary artery stenosis. CSF phenomenon presents with various manifestations like myocardial ischemia, life-threatening arrhythmias, sudden cardiac death, and recurrent acute coronary syndromes (ACS). However, the etiopathogenesis of CSF phenomenon remains only partially understood. Endothelial dysfunction, subclinical atherosclerosis,

(C)2019 Women in Cardiology and Related Sciences
License terms

$($ (1) $\odot \circledast$ 
inflammation, ectasia, small vessel disease, and heart failure are suggested as the various pathophysiological factors of CSF

This study aims to correlate risk factors, clinical profile, laboratory findings, and angiographic profile and find out the common predictors in patients with CSF.

\section{Material and Methods}

The case-control study included patients who underwent coronary angiography between June 1, 2017, and May 31,2018 , in our institute were evaluated. A total of 100 consecutive patients, cases with CSF phenomenon and 100 controls with normal coronary (NC) flow were evaluated in the present study.

\section{Cases: CSF}

\section{Inclusion Criteria}

Patients older than 18 years who present with chest pain or symptoms suggestive of angina equivalent with normal epicardial coronaries on coronary angiography but with the CSF in any of the coronary arteries are studied.

\section{Exclusion Criteria}

Exclusion criteria included coronary artery disease (plaque, obstructive lesion), causes of secondary CSF phenomenon (spasm, ectasia), myocardial bridging, valvular disease, and left ventricular systolic dysfunction.

\section{Control Group}

The control group (NC) comprised of 100 patients who underwent coronary angiography, which showed normal epicardial coronary artery and normal flow.

Risk factors (age, sex, diabetes, hypertension, dyslipidemia, body mass index [BMI], and smoking), hematology and biochemical parameters like complete blood picture, total platelet count, total and differential leucocyte count, platelet-to-lymphocyte ratio (PLR) and neutrophil-to-lymphocyte ratio (NLR), uric acid, lipid profile, high-sensitivity C-reactive protein (Hs-CRP), and HBA1C were assessed. In both groups, demographic and clinical information was collected, and laboratory parameters were measured and compared.

Coronary blood flow was measured by using corrected thrombolysis in myocardial infarction (TIMI) frame count (CTFC). The slow flow was defined as CTFC beyond 2 standard deviations (SDs) from normal published range.

\section{Coronary Angiogram}

Coronary angiogram was performed using optitorque angiographic catheters with manual injection. The angiograms for coronary flow measurement was studied using the CTFC method and the count value which exceeds 27 , that is, greater than 2 SDs from the normal published range of $21 \pm 3$ was noted as abnormal and suggestive of CSF phenomenon as described by Gibson et al. ${ }^{1}$ Image frame count of
15 frames/second, and a correction factor of 1.7 for the left anterior descending (LAD) was taken for study.

\section{Statistical Results}

Chi-square/Fisher's exact test was studied to compare the statistical significance of categorical variables. Statistical significance of the quantitative variables was analyzed with unpaired Student's $t$-test. The equality of variances between the two groups was studied by Leven's test. Mann Whitney $\mathrm{U}$ test was applied when quantitative variables did not follow a normal distribution. Multivariable logistic regression was applied by using the enter method, and variables which had a p-value of less than 0.05 by univariable methods were included. The statistical software Minitab 17 (Minitab, Ltd., United Kingdom) was used to analyze the data.

\section{Results}

This study consists of 100 cases (CSF) and 100 controls (NC) which include $66 \%$ and $58 \%$ of males, $34 \%$ and $42 \%$ of females in case and control groups, respectively ( - Table 1 ).

Mean age of case group is $54.97 \pm 10.9$ years and control group is $55.17 \pm 10.8$ years. Hypertension is present in $66 \%$ and $38 \%$, DM in $67 \%$ and $37 \%, 46 \%$ and $14 \%$ are smokers, $43 \%$ and $19 \%$ are alcoholic, and dyslipidemia is present in $65 \%$ and $36 \%$ in case and control groups, respectively (-Tables 2 and $\mathbf{3}$; - Figs. 1-5).

Various laboratory parameters like hematocrit, platelet count, platelet-to-lymphocyte ratio (PLR) and neutrophil-to-lymphocyte ratio (NLR), uric acid, total cholesterol, triglycerides, and Hs-CRP were assessed. Inflammatory markers like uric acid ( $p=0.03)$ and Hs-CRP $(p=0.000)$ were found to be statistically significant. Hematocrit $(p=0.023)$, NLR $(p=0.001)$ total cholesterol $(p=0.000)$, triglycerides $(p=0.000)$, and BMI $(p=0.000)$ were statistically significant. PLR has the tendency of statistically significant $(p=0.059)$ ( - Table 4$)$.

LAD $(n=50)$ is most frequently involved coronary artery followed by left circumflex artery (LCX) $(n=16))$ and RCA $(n=34)$, and right dominant circulation noted in $89 \%$ in case group (CSF) (-Table 5; - Figs. 6 and $\mathbf{7}$ ).

The most common presentation was chronic stable angina (CSA) in 56\% and the remaining $44 \%$ with the acute coronary syndrome (ACS) (28\% with the unstable angina [USA] and $16 \%$ with non-ST elevated myocardial infarction [NSTEMI]) in patients with CSF ( - Fig. 8).

\section{Discussion}

This study analyzed the laboratory predictors, risk factors, clinical presentation, and angiographic profile of patients associated with CSF phenomenon. In our study, BMI is a strongly associated factor $(p=0.000)$ with CSF, which was also similar to other studies reported by authors. ${ }^{2-4}$ Yilmaz et $^{2}{ }^{2}$ in

Table 1 Demographic data

\begin{tabular}{|l|l|l|}
\hline Variable & $\begin{array}{l}\text { Case (CSF) } \\
(\boldsymbol{n}=100)\end{array}$ & $\begin{array}{l}\text { Control (NC) } \\
(\boldsymbol{n}=\mathbf{1 0 0})\end{array}$ \\
\hline Male & 66 & 58 \\
\hline Female & 34 & 42 \\
\hline
\end{tabular}


Table 2 Risk factors among the study groups in males

\begin{tabular}{|l|l|l|l|}
\hline Males & Cases (CSF) $n=66$ & Control (NC) $n=58$ & $p$-Value \\
\hline Hypertension & $43(65.1 \%)$ & $22(37.9 \%)$ & 0.002 \\
\hline Diabetes mellitus & $48(72.7 \%)$ & $27(46.5 \%)$ & 0.002 \\
\hline Smoking & $45(68.1 \%)$ & $14(24.1 \%)$ & 0.000 \\
\hline Alcohol & $42(63.6 \%)$ & $19(32.7 \%)$ & 0.000 \\
\hline Dyslipidemia & $43(65.1 \%)$ & $23(39.6 \%)$ & 0.003 \\
\hline
\end{tabular}

Table 3 Risk factors among the study groups in Females

\begin{tabular}{|l|l|l|l|}
\hline Females & Cases (CSF) $\boldsymbol{n}=34$ & Control (NC) $\boldsymbol{n}=\mathbf{4 2}$ & $p$-Value \\
\hline Hypertension & $23(67.4 \%)$ & $16(38.1 \%)$ & 0.007 \\
\hline Diabetes mellitus & $19(55.9 \%)$ & $10(23.8 \%)$ & 0.003 \\
\hline Smoking & $01(2.9 \%)$ & 00 & - \\
\hline Alcohol & $01(2.9 \%)$ & 00 & - \\
\hline Dyslipidemia & $22(67.6 \%)$ & $13(30.9 \%)$ & 0.001 \\
\hline
\end{tabular}
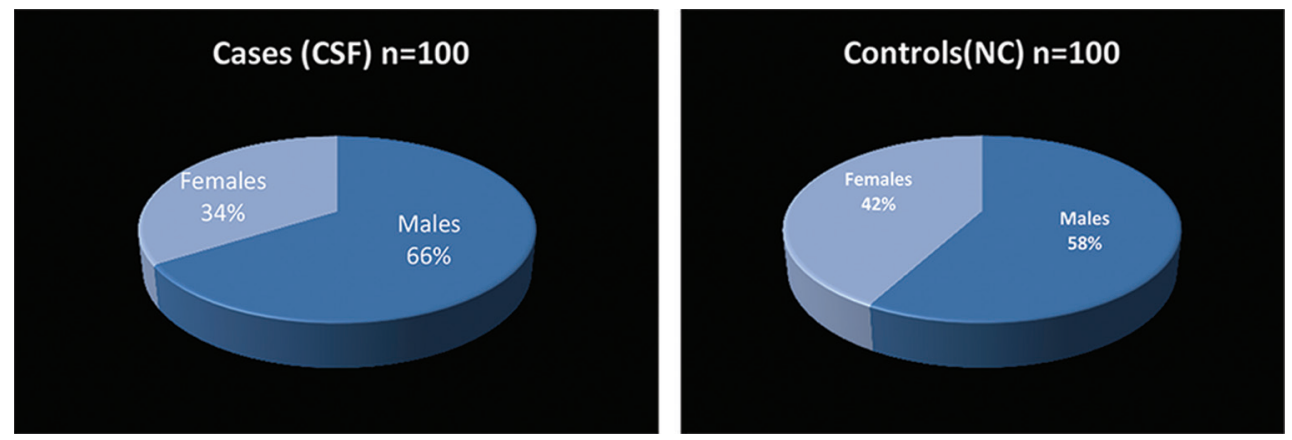

Fig. 1 Gender distribution between study groups.

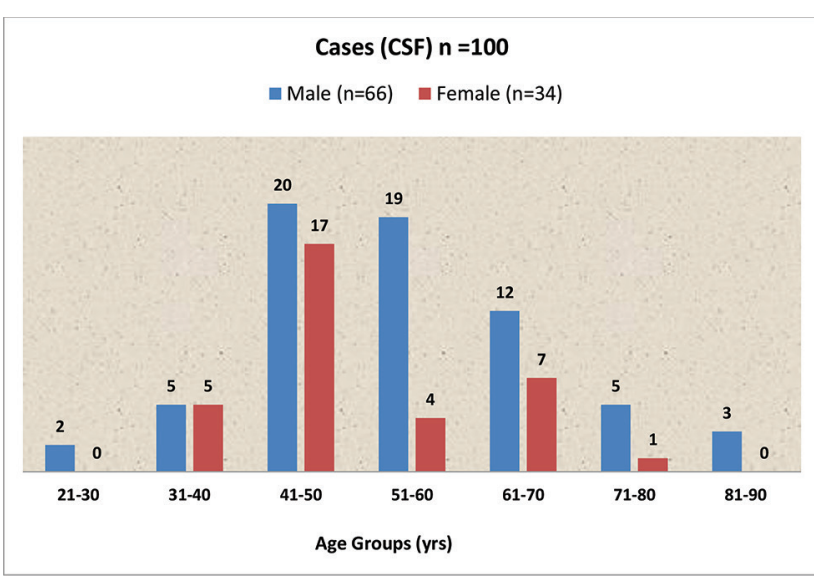

Fig. 2 Age distribution in cases (CSF) group.

a Turkish population study and Hawkins et $\mathrm{al}^{4}$ in the North American population showed BMI to have an independent association with CSF. In another study performed by Beltrame et al in an Australian population, males and smokers were independent risk factors for CSF. ${ }^{5}$ A study in Chinese population showed that hyperglycemia, Hs-CRP, and increased platelet count and uric acid which causes endothelial dysfunction are the risk factors for $\mathrm{CSF}^{6}$ which is similar to our

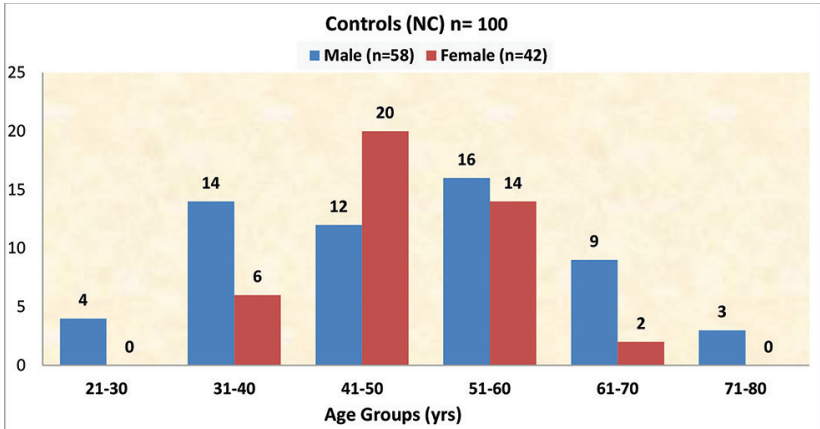

Fig. 3 Age distribution in control (NC) group.

study population. A study in an Iranian population showed that diabetes and hypertension are independent risk factors for CSF, which is similar to our study group. Because of multiple causative factors for slow flow, the evidence shows the endothelial dysfunction, which causes small vessel disease is the main causative factor responsible for CSF phenomenon.

In our study, the common clinical presentation was chronic stable angina (56\%) and 44\% with ACS (28\% with USA), and $16 \%$ with (NSTEMI in patients with CSF. Presentation is diverse from atypical chest pain, chronic stable angina or USA, NSTEMI to ST-elevation myocardial infarction (STEMI) 


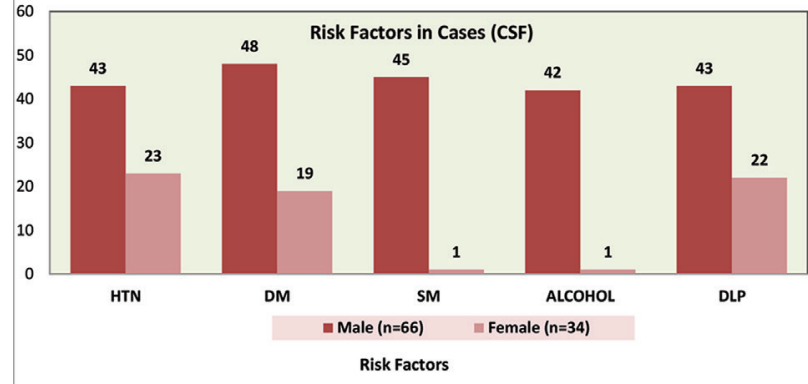

Fig. 4 Risk factors in cases (CSF) group.

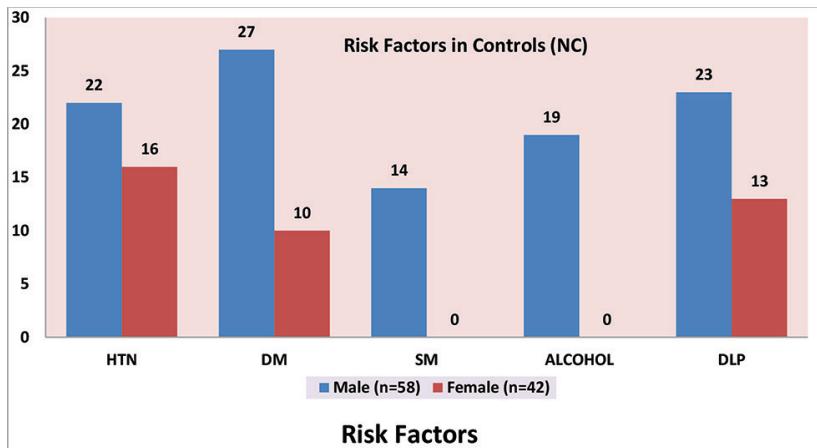

Fig. 5 Risk factors in control (NC) group.

Table 4 Laboratory variables in case and control

\begin{tabular}{|l|l|l|l|}
\hline Variable & Case (CSF) & Control (NC) & $P$-Value \\
\hline Hematocrit $(\%)$ & $42.5 \pm 3.17$ & $41.5 \pm 3.01$ & 0.023 \\
\hline Platelet count $\left(\mathrm{lakh} / \mathrm{mm}^{3}\right)$ & $2.06 \pm 0.59$ & $2.01 \pm 0.69$ & 0.582 \\
\hline PLR $(\%)$ & $2.4 \pm 1.3$ & $2.1 \pm 0.9$ & 0.059 \\
\hline NLR $(\%)$ & $3 \pm 1.8$ & $2.3 \pm 1.2$ & 0.001 \\
\hline Uric acid $(\mu \mathrm{mol} / \mathrm{L})$ & $6.19 \pm 1.73$ & $5.7 \pm 1.42$ & 0.03 \\
\hline Hs-CRP $(\mathrm{mg} / \mathrm{L})$ & $11.3 \pm 1.6$ & $6.5 \pm 1.1$ & 0.000 \\
\hline Total cholesterol $(\mathrm{mg} / \mathrm{dL})$ & $216 \pm 44.1$ & $148.8 \pm 18.8$ & 0.000 \\
\hline Triglycerides $(\mathrm{mg} / \mathrm{dL})$ & $206.1 \pm 71.3$ & $116.3 \pm 16.1$ & 0.000 \\
\hline BMI & $32.4 \pm 4.2$ & $23 \pm 1.5$ & 0.000 \\
\hline
\end{tabular}

Abbreviations: PLR, platelet-to-lymphocyte ratio; NLR, neutrophil-to-lymphocyte ratio; Hs-CRP, high-sensitivity C-reactive protein.

Table 5 Slow flow distribution in coronary vessels

\begin{tabular}{|l|l|l|l|l|l|}
\hline \multirow{2}{*}{} & \multicolumn{3}{|c|}{ Slow flow } & \multicolumn{2}{c|}{ Dominance } \\
\cline { 2 - 6 } & LAD & LCX & RCA & Right & Left \\
\hline Males & 34 & 11 & 21 & 57 & 09 \\
\hline Females & 16 & 05 & 13 & 32 & 02 \\
\hline
\end{tabular}

Abbreviations: LAD, left anterior descending; LCX, left circumflex artery; RCA, right coronary artery.

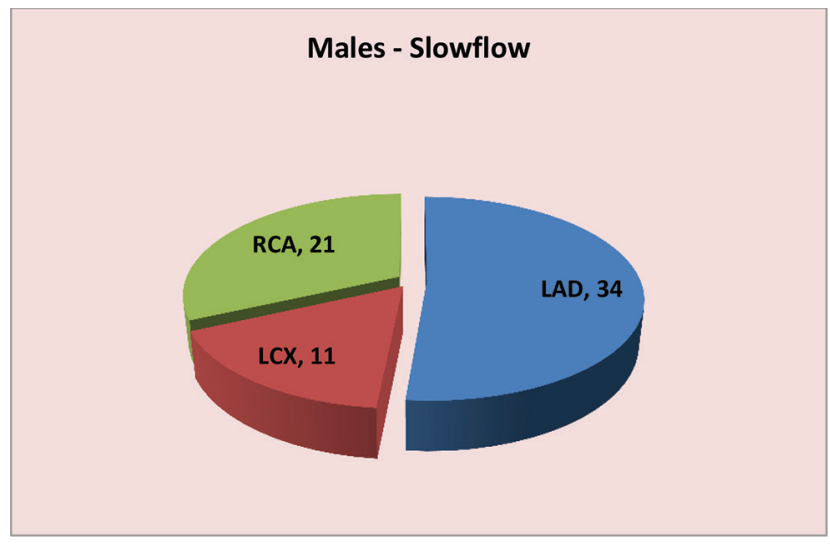

Fig. 6 Slow flow distribution in coronary vessels (males).

as studies by Gökçe et al and Amirzadegan et al $\mathrm{l}^{5,7-10}$ show. In a study conducted in the Iranian population with CSF phenomenon, $75 \%$ of the patients presented with ACS which is different from our study where CSA is more common. The most common presentation of CSF phenomenon was

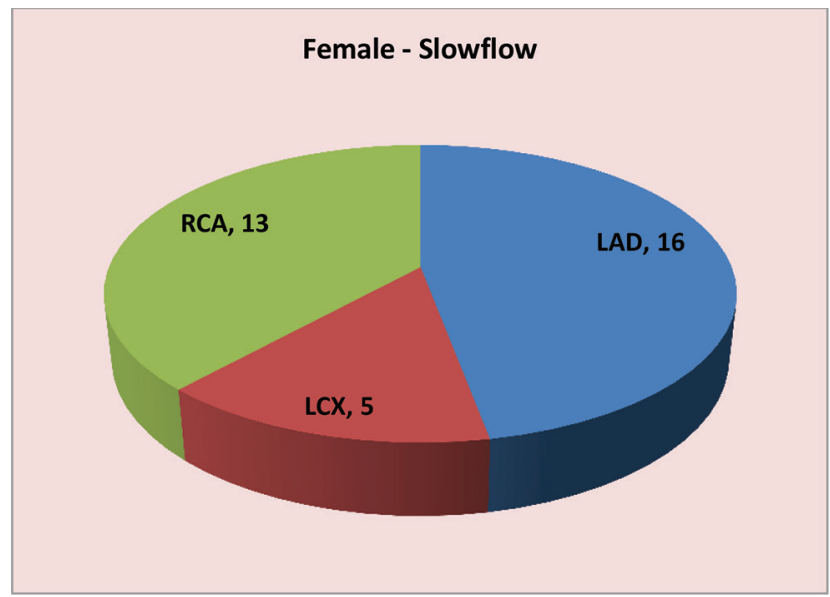

Fig. 7 Slow flow distribution in coronary vessels (females).

nonspecific chest pain (71.9\%) followed by ACS (18.4\%) and chronic stable angina ( $8.8 \%$ ) in a study conducted by Yaron et $\mathrm{al}^{11}$ in an Israeli population. LAD artery is the most common 


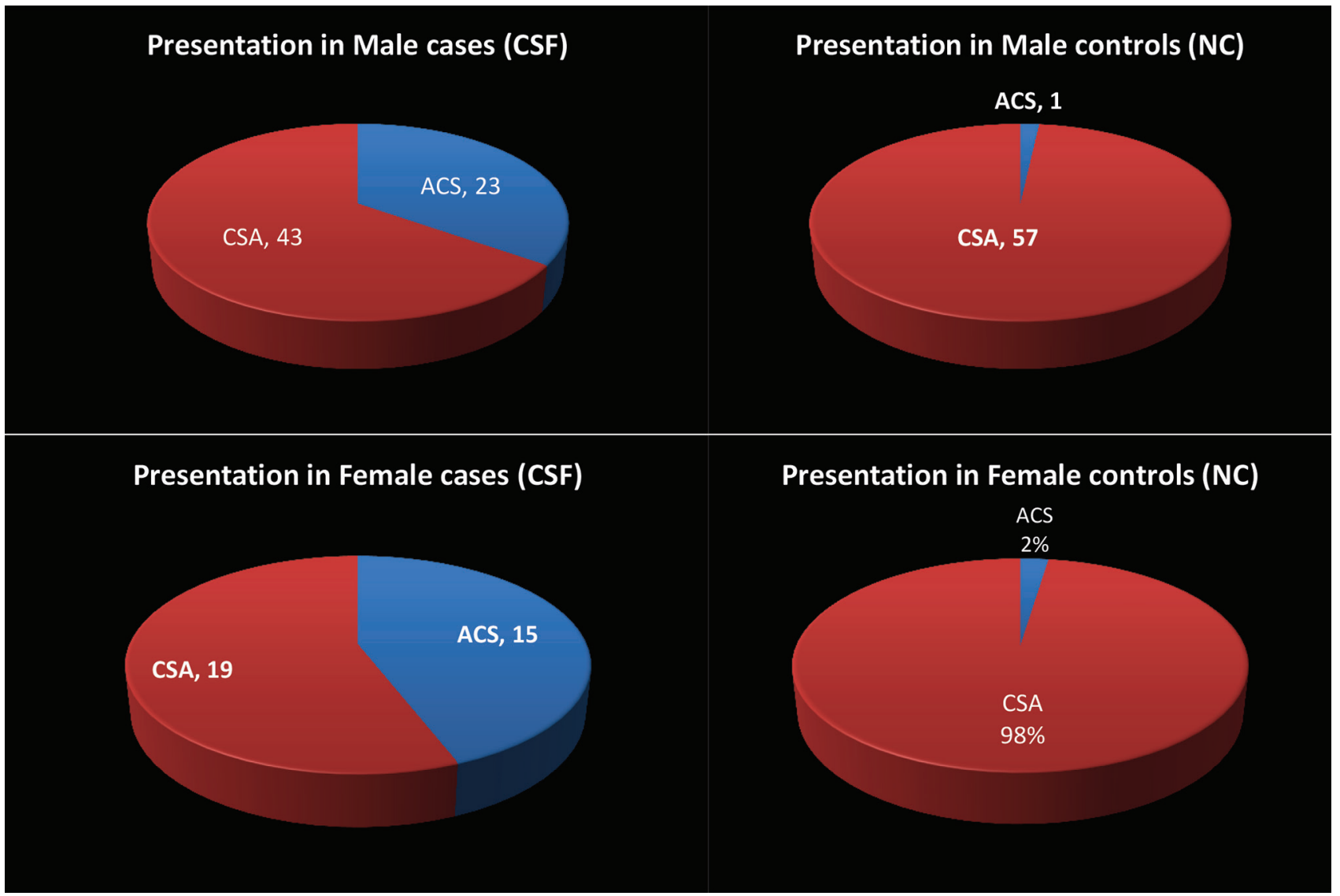

Fig. 8 Gender distribution of presentation between study groups.

coronary artery involved $(n=50)$, followed by LCX $(n=16)$ and right coronary artery (RCA) $(n=34)$ which is similarly reported in other study populations. ${ }^{8,10}$ Pontiroli et $\mathrm{al}^{12}$ in his study population showed that a reduction in BMI after surgical procedures like gastric banding is associated with significant improvement in markers causing endothelial dysfunction, which is the causative factor for CSF phenomenon. BMI is found to be the strongest predictor of coronary slow flow $(p=0.000)$ in our study population. Newer studies need to be conducted in the future to see whether a reduction in BMI decreases the markers of endothelial dysfunction, which causes CSF phenomenon and improves the coronary flow.

The endothelium plays a pivotal role by regulating coronary vascular tone and control coronary blood flow. ${ }^{13}$ Measurement of endothelial function, by the brachial artery flow-mediated dilatation (FMD), is impaired in people presenting with $\mathrm{CSF}^{14}$ In patients with CSF nitric oxide (NO) bioactivity is decreased, and impaired vascular endothelial function is seen. ${ }^{15}$ It is noted that the concentration of adiponectin and paraoxonase activity two critical markers of endothelial dysfunction are markedly reduced in patients with a CSF. ${ }^{16}$ High levels of serum uric acid were found in patients with endothelial dysfunction, which is a causative factor for CSF phenomenon. ${ }^{17}$ Uric acid is known to induce endothelial dysfunction via down-regulating NO production and by mitochondrial $\mathrm{Na}^{+} / \mathrm{Ca}^{2+}$ exchanger-mediated mitochondrial calcium overload. ${ }^{18}$ Besides, Elbasan et $\mathrm{al}^{19}$ in their study in cardiac syndrome $\mathrm{X}$ patients demonstrated a significant positive independent association between levels of serum uric acid and CSF phenomenon. In our study, we found that serum uric acid levels were significantly higher in patients with CSF phenomenon when compared with the control group. There is considerable evidence that CSF occurs as a result of coronary microvascular dysfunction, which is secondary to endothelial dysfunction. It was noted that coronary flow reserve (CFR), as an indicator of coronary microvascular function, is impaired in patients with CSF phenomenon. ${ }^{20}$ Reduced CFR is the earliest manifestation of coronary atherosclerosis. Kanbay et $\mathrm{al}^{21}$ showed that uric acid has a role in coronary microvascular disease and CFR. Uric acid by inducing vascular smooth muscle cell proliferation and causing increased oxidative stress via the vascular renin-angiotensin system causes coronary microvascular disease. ${ }^{22}$ Güllü et al $^{23}$ have found that serum uric acid levels are inversely related with CFR which have a role in coronary microvascular disease in healthy individuals. Cin et $\mathrm{al}^{24}$ investigated coronary artery morphology in patients with CSF phenomenon and found out that diffuse intimal thickening, atheroma, does not cause luminal irregularities and widespread calcification in the coronary vessel wall, suggesting that the CSF phenomenon is a form of early-stage coronary atherosclerosis in their coronary angiographic analysis of patients with CSF. Consistent with our results, Naing et al recently found that uric acid was the most important determining risk factor for CSF phenomenon.

Inflammation has a vital role in the pathogenesis of CSF; the association of CSF with inflammatory markers remains 
controversial. Association of inflammation with CSF is noted in various studies. ${ }^{25}$ NLR ratio provides an easy but also a promising method to screen for systemic inflammation, and it is widely used as a marker for CSF cardiovascular diseases. ${ }^{26}$ Elevated NLR and serum uric acid is indicative of a systemic inflammatory response and was found to be associated with $\operatorname{CSF}^{27,28}$ and statistically significant $(p=0.001)$. In some studies, elevated hemoglobin or hematocrit is noted in patients with $\mathrm{CSF}^{29,30}$ which does not have statistical significance as noted in other studies. ${ }^{31,32}$ In our study, hematocrit was statistically significant $(p=0.002)$; Cetin et al showed blood viscosity, which is based on hematocrit and total serum protein, is an independent predictor of CSF. ${ }^{33}$ Endothelial dysfunction is induced by increased blood viscosity in patients with CSF phenomenon. Platelet activation is precipitated by increased blood viscosity and shear stress in coronary arteries. ${ }^{34}$ In recent studies, PLR is found to be higher in patients with $\mathrm{CSF}^{35,36} \mathrm{PLR}$ is raised in CSF cases and has a tendency for being statistically significant $(p=0.059)$.

Earlier studies have shown that endothelial dysfunction, subclinical atherosclerosis, inflammation, small vessel disease, and anatomy of coronary arteries are the causative factors for CSF phenomenon. The current findings showed that CSF is a part of systemic vascular disorder and inflammation.

Further studies are needed to reveal the pathogenesis causing CSF. Large-scale clinical studies are necessary to characterize the CSF phenomenon better and investigate various potential therapeutic approaches.

\section{Limitations}

The design of our study is descriptive and retrospective. A single-spot blood sample was taken, and the temporal trend of changes during hospitalization was not known.

\section{Conclusions}

CSF was common in males, smokers, and DM patients and is associated with high NLR, uric acid, and Hs-CRP levels. We found BMI, Hs-CRP, total cholesterol, and triglycerides to have an independent risk factor associated with CSF phenomenon in our study. These findings provide an additional impetus for further studies to confirm the role of other inflammatory markers in CSF patients and treatment strategies depending on that.

\section{Conflict of Interest}

None.

\section{References}

1 Gibson CM, Cannon CP, Daley WL, et al. TIMI frame count: a quantitative method of assessing coronary artery flow. Circulation 1996;93(5):879-888

2 Yilmaz H, Demir I, Uyar Z. Clinical and coronary angiographic characteristics of patients with coronary slow flow. Acta Cardiol 2008;63(5):579-584

3 Chaudhry MA, Smith M, Hanna EB, Lazzara R. Diverse spectrum of presentation of coronary slow flow phenomenon: a concise review of the literature. Cardiol Res Pract 2012;2012:383181
4 Hawkins BM, Stavrakis S, Rousan TA, Abu-Fadel M, Schechter E. Coronary slow flow-prevalence and clinical correlations. Circ J 2012;76(4):936-942

5 Beltrame JF, Limaye SB, Horowitz JD. The coronary slow flow phenomenon-a new coronary microvascular disorder. Cardiology 2002;97(4):197-202

6 Xia S, Deng SB, Wang Y, et al. Clinical analysis of the risk factors of slow coronary flow. Heart Vessels 2011;26(5):480-486

7 Moazenzadeh M, Azimzadeh BS, Zare J, et al. Clinical features and main determinants of coronary slow flow phenomenon in Iranian patients. Eur J Cardiovasc Med 2010;1(2):2042-4884

8 Gökçe M, Kaplan S, Tekelioğlu Y, Erdoğan T, Küçükosmanoğlu M. Platelet function disorder in patients with coronary slow flow. Clin Cardiol 2005;28(3):145-148

9 Amirzadegan A, Motamed A, Davarpasand T, Shahrzad M, Lotfi-Tokaldany $\mathrm{M}$. Clinical characteristics and mid-term outcome of patients with slow coronary flow. Acta Cardiol 2012;67(5):583-587

10 Sanati H, Kiani R, Shakerian F, et al. Coronary slow flow phenomenon: clinical findings and predictors. Res Cardiovasc Med 2016;5(1):e30296

11 Arbel Y, Rind E, Banai S, et al. Prevalence and predictors of slow flow in angiographically normal coronary arteries. Clin Hemorheol Microcirc 2012;52(1):5-14

12 Pontiroli AE, Pizzocri P, Paroni R, Folli F. Sympathetic overactivity, endothelial dysfunction, inflammation, and metabolic abnormalities cluster in grade III (World Health Organization) obesity: reversal through sustained weight loss obtained with laparoscopic adjustable gastric banding. Diabetes Care 2006;29(12):2735-2738

13 Lüscher TF, Richard V, Tschudi M, Yang ZH, Boulanger C. Endothelial control of vascular tone in large and small coronary arteries. J Am Coll Cardiol 1990;15(3):519-527

14 Damaske A, Muxel S, Fasola F, et al. Peripheral hemorheological and vascular correlates of coronary blood flow. Clin Hemorheol Microcirc 2011;49(1-4):261-269

15 Sezgin N, Barutcu I, Sezgin AT, et al. Plasma nitric oxide level and its role in slow coronary flow phenomenon. Int Heart J 2005;46(3):373-382

16 Selcuk H, Selcuk MT, Temizhan A, et al. Decreased plasma concentrations of adiponectin in patients with slow coronary flow. Heart Vessels 2009;24(1):1-7

17 Kanbay M, Segal M, Afsar B, Kang DH, Rodriguez-Iturbe B, Johnson RJ. The role of uric acid in the pathogenesis of human cardiovascular disease. Heart 2013;99(11):759-766

18 Papežíková I, Pekarová M, Kolářová H, et al. Uric acid modulates vascular endothelial function through the down regulation of nitric oxide production. Free Radic Res 2013;47(2):82-88

19 Elbasan Z, Sahin DY, Gür M, et al.Serum uric acid and slow coronary flow in cardiac syndrome X. Herz 2013;38(5):544-548

20 Erdogan D, Caliskan M, Gullu H, Sezgin AT, Yildirir A, Muderrisoglu $\mathrm{H}$. Coronary flow reserve is impaired in patients with slow coronary flow. Atherosclerosis 2007;191(1):168-174

21 Kanbay M, Sánchez-Lozada LG, Franco M, et al. Microvascular disease and its role in the brain and cardiovascular system: a potential role for uric acid as a cardiorenal toxin. Nephrol Dial Transplant 2011;26(2):430-437

22 Corry DB, Eslami P, Yamamoto K, Nyby MD, Makino H, Tuck ML. Uric acid stimulates vascular smooth muscle cell proliferation and oxidative stress via the vascular renin-angiotensin system. J Hypertens 2008;26(2):269-275

23 Gullu H, Erdogan D, Caliskan M, et al. Elevated serum uric acid levels impair coronary microvascular function in patients with idiopathic dilated cardiomyopathy. Eur J Heart Fail 2007;9(5):466-468

24 Cin VG, Pekdemir H, Camsar A, et al. Diffuse intimal thickening of coronary arteries in slow coronary flow. Jpn Heart J 2003;44(6):907-919 
25 Barutcu I, Sezgin AT, Sezgin N, et al.Increased high sensitive CRP level and its significance in pathogenesis of slow coronary flow. Angiology 2007;58(4):401-407

26 Arbel Y, Finkelstein A, Halkin A, et al. Neutrophil/lymphocyte ratio is related to the severity of coronary artery disease and clinical outcome in patients undergoing angiography. Atherosclerosis 2012;225(2):456-460

27 Cingoz F, Iyisoy A, Demirkol S, et al. Carotid intima-media thickness in patients with slow coronary flow and its association with neutrophil-to-lymphocyte ratio: a preliminary report. Clin Appl Thromb Hemost 2014;20(4):393-399

28 Lyngdoh T, Marques-Vidal P, Paccaud F, et al. Elevated serum uric acid is associated with high circulating inflammatory cytokines in the population-based Colaus study. PLoS One 2011;6(5):e19901

29 Altun I, Akin F, Kose N, Sahin C, Kirli I. Predictors of slow flow in angiographically normal coronary arteries. Int J Clin Exp Med 2015;8(8):13762-13768

30 Zalawadiya SK, Veeranna V, Niraj A, Pradhan J, Afonso L. Red cell distribution width and risk of coronary heart disease events. Am J Cardiol 2010;106(7):988-993

31 Patel KV, Mohanty JG, Kanapuru B, Hesdorffer C, Ershler WB, Rifkind JM. Association of the red cell distribution width with red blood cell deformability. Adv Exp Med Biol 2013;765:211-216

32 Vecchio S, Varani E, Chechi T, et al. Coronary thrombus in patients undergoing primary PCI for STEMI: Prognostic significance and management. World J Cardiol 2014;6(6):381-392

33 Cetin MS, Ozcan Cetin EH, Canpolat U, et al. Overlookeded parameter in coronary slow flow phenomenon: whole blood viscosity Biomark Med 2015;9:1311-1321

34 Shankaran H, Alexandridis P, Neelamegham S. Aspects of hydrodynamic shear regulating shear-induced platelet activation and self-association of von Willebrand factor in suspension. Blood 2003;101(7):2637-2645

35 Akboga MK, Canpolat U, Balci KG, et al. Increased platelet to lymphocyte ratio is related to slow coronary flow. Angiology 2016;67(1):21-26

36 Oylumlu M, Doğan A, Oylumlu M, et al. Relationship between platelet-to-lymphocyte ratio and coronary slow flow. Anatol J Cardiol 2015;15(5):391-395 